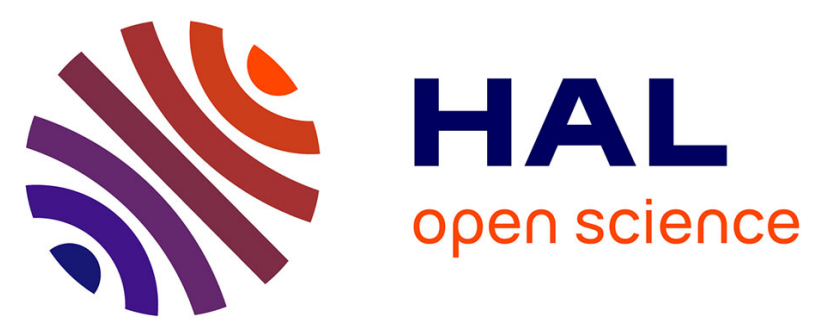

\title{
Multi-axis MEMS force sensor for measuring friction components involved in dexterous micromanipulation: Design and optimization.
}

\author{
Margot Billot, Xin Xu, Joël Agnus, Emmanuel Piat, Philippe Stempflé
}

\section{To cite this version:}

Margot Billot, Xin Xu, Joël Agnus, Emmanuel Piat, Philippe Stempflé. Multi-axis MEMS force sensor for measuring friction components involved in dexterous micromanipulation: Design and optimization.. International Journal of Nanomanufacturing, 2015, 11 (3/4), pp.161-184. 10.1504/IJNM.2015.071924 . hal-01303538

\section{HAL Id: hal-01303538 \\ https://hal.science/hal-01303538}

Submitted on 28 Apr 2016

HAL is a multi-disciplinary open access archive for the deposit and dissemination of scientific research documents, whether they are published or not. The documents may come from teaching and research institutions in France or abroad, or from public or private research centers.
L'archive ouverte pluridisciplinaire HAL, est destinée au dépôt et à la diffusion de documents scientifiques de niveau recherche, publiés ou non, émanant des établissements d'enseignement et de recherche français ou étrangers, des laboratoires publics ou privés. 


\title{
Multi-axis MEMS force sensor for measuring friction components involved in dexterous micromanipulation: Design and optimization
}

\section{Margot Billot, Xin Xu, Joël Agnus, Emmanuel Piat* and Philippe Stempflé}

\author{
FEMTO-ST Institute \\ Univ. Bourgogne Franche-Comté, Univ. de Franche- \\ Comté/CNRS/ENSMM/UTBM, \\ 24 rue Savary, F-25000 Besançon, FRANCE \\ E-mails: margot.billot@femto-st.fr, xin.xu@femto-st.fr, \\ joel.agnus@ens2m.fr, emmanuel.piat@ens2m.fr, \\ philippe.stempfle@ens2m.fr \\ ${ }^{*}$ Corresponding author
}

\begin{abstract}
At the nanoscale and for particular applications such as dexterous micro-manipulation, two Degrees of Freedom nanotribometers are no longer adequate for studying and characterizing the contacts. This paper deals with the specifications and working principle of a new multi-axis friction sensor designed for nanotribological testing applied to this purpose in order to extract each contribution independently (ie, sliding, rolling and spin motion). It is composed of a central platform with a fixed ball and surrounded by a compliant table. Its sensing ability is based on piezoresistivity: four sets of piezoresistors are symmetrically distributed at the root of four central beams. Finite Elements Method simulations are performed to find the optimal dimensions of the sensor. As results, this sensor could measure independently normal and friction forces in the range of $1 \mathrm{mN}$ and $100 \mu \mathrm{N}$, respectively and the three rotation components. Estimated crosstalk is lower than $1 \%$ with a good sensitivity.
\end{abstract}

Keywords: MEMS force sensor; Nanotribology; Multi-axis force sensing; Dexterous micro-manipulation; Friction; Piezoresistive sensor; Finite Elements Method simulation

\section{Introduction}

In assembly of micro-components below $1 \mathrm{~mm}$, two approaches are currently considered [1]: (i) the self-assembly paradigm [2, 3, 4] in which surface effects are used to organize and assemble structures mainly up to a few micrometers, and (ii) the micro-robotic assembly [1, 5, 6, 7] that is well suitable when the main challenging issues concern the handling and assembly of small components - as met for instance in some specific hybrid MEMS [8, 9, 10]. The latter approach is generally based on high resolution micro-manipulators and 
Figure 1 In classical microgripping: any rotation of the object (eg, transition a-c) needs a global rotation of the manipulator (rotations $a-b$ and $b-c$ ).

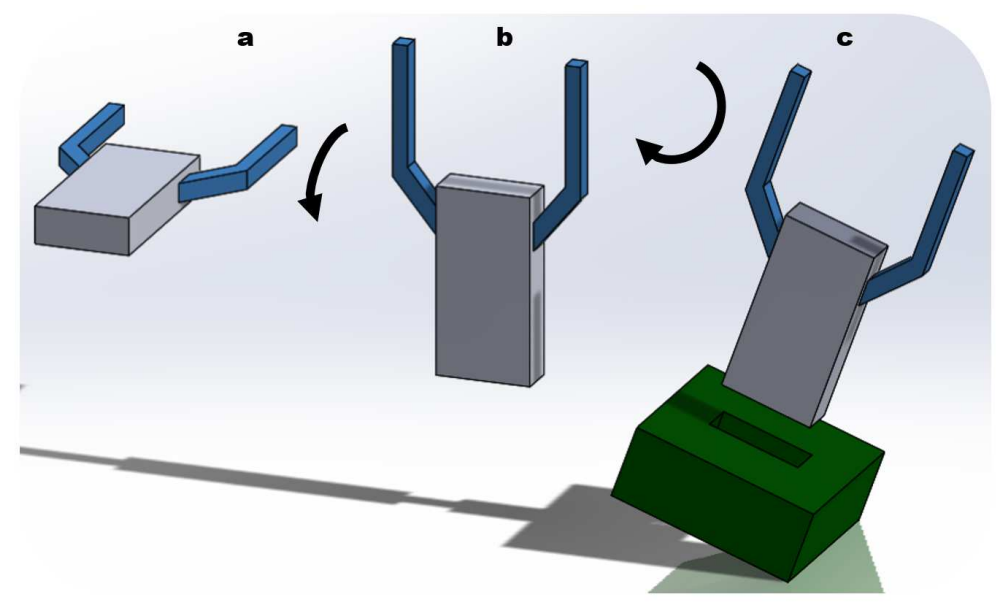

gripping devices with end-effectors controlled in position and more recently in force. Hence, the development of micro-grippers that can accurately perform both translation and rotation positioning of the manipulated object appears as a real challenge for the industrial field of micro-assembly. Owing to the difficulties of designing micro-grippers that perform accurate angular positioning, current industrial solutions generally favour to rotate the whole robotic system - including the gripper - instead of the manipulated object, as shown in Fig. 1. Indeed, transition from the position (1.a) to the position (1.c) involves two rotations of the grippers (transitions $\mathrm{a}-\mathrm{b}$ and $\mathrm{b}-\mathrm{c}$ ) displaying a poor accuracy of positioning. However, another recent approach consists in performing local dexterous micro-manipulation of the object [11, 12] in order to improve the positioning ability. As suggested in Fig. 2, the main advantage of this approach is that each rotation of the object can be locally induced by accurate translations (transitions a-b and c-d) of the fingers only. As a result, the micro-manipulation performance can be improved because translation devices display finer positioning and more compact system with less stages for at least the same number of Degrees of Freedom (DOF). However as a drawback, dexterous manipulation requires an accurate control of the forces that are applied to the object, which are themselves intimately connected to the contact that is involved between the object and the fingers. Indeed, as shown in Fig. 3 , translation movements of fingers can induce, at the interface between the object and either the finger's edge or finger's apex, various frictional components: ie, sliding (3a), rolling (3b) or spin motion (3c). Besides, each kind of contact is likely to change, in turn, the applied contact forces during the process. It appears that these tribological components are essential to move the object by dexterous manipulation. Hence, the contact between fingers and object has to be known and controlled in real time, meaning that their frictional and adhesion behaviours have to be mastered for each kind of movements inducing sliding, rolling and spin motion components simultaneously.

Nanotribological approach was recently used for assessing frictional components during quasi-static and dynamic micro-gripping process [13]. Indeed, a ball-on-disc nanotribometer was used for simulating a micro-gripper that grabs a spherical object. This approach was well suitable for studying the sliding component but is clearly inadequate for assessing several frictional components simultaneously. Among the other existing tribometers [14, 15, 16, 17], 
Figure 2 In dexterous manipulation, the same movement of the object (eg, transition a-d) only needs fingers translation (translations a-b and c-d). No further rotation movement is required. The micro-manipulation system can be simpler, more compact and accurate.

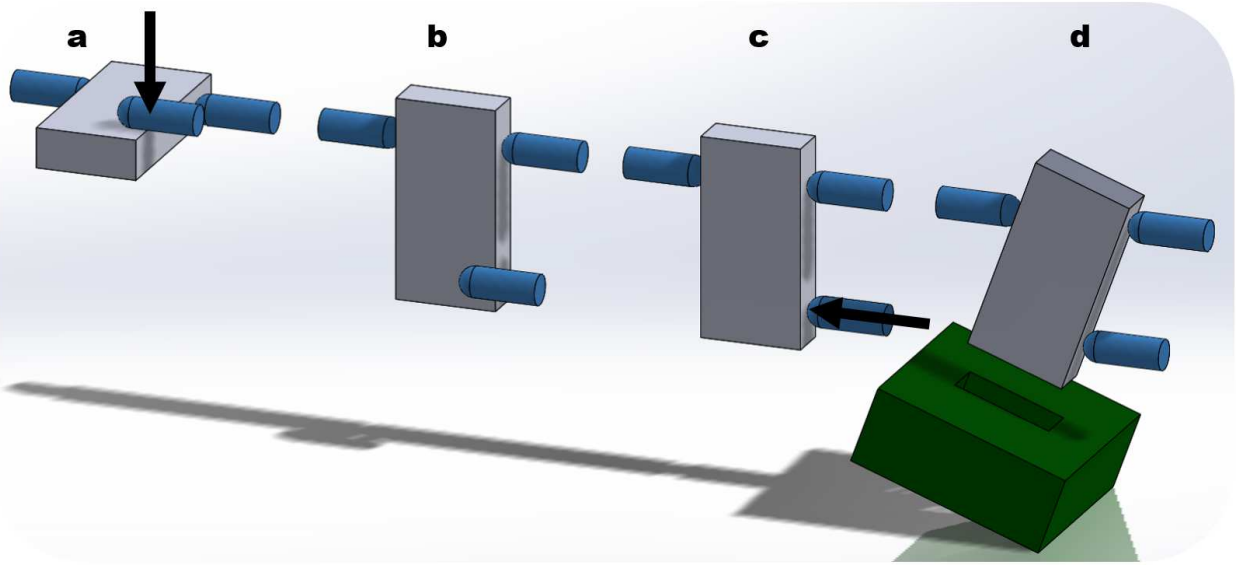

Figure 3 Different contacts between finger and manipulated object that are likely to occur during dexterous manipulation : a) sliding of finger's apex and finger's edge ; b) rolling around the finger's apex and finger's edge ; c) spin motion around the finger's apex.

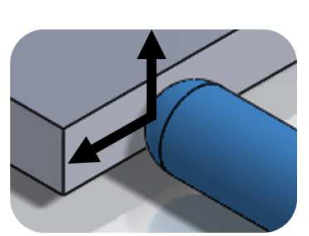

a

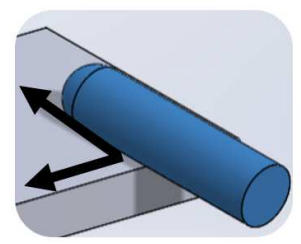

b
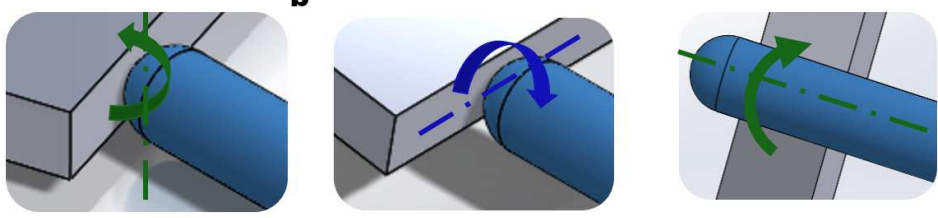

c

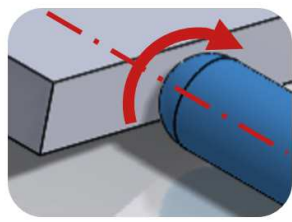


the Tribolever proposed by Zijlstra [18] is well designed to characterize sliding components. It is composed of a central detection pyramid surrounded by four high aspect ratio legs. Lateral or frictional forces acting on the scanning tip are measured via the displacement of the pyramid by means of four laser interferometers [18]. This structure is able to measure the 3 translation components with theoretically no crosstalk. But unfortunately, it was designed in order to limit torsional movements, so it cannot be suitable for measuring the rolling and spin motion component of friction. Hence, a multi-axis device with low crosstalks is then needed for this purpose.

The aim of this paper is to design a new type of nanotribometer enabling to simulate the finger/object contact. It has to display a flexible structure which allows torsion moves in order to access to all the components of friction simultaneously. The combination of this compliant structure with a piezoresistive sensing technology [19, 20] allows to obtain a bulk multi-axis nanotribometer characterized by high displacement, high sensitivities and acceptable crosstalks. Section 2 presents the specifications and working principle of this new sensor. Its design and behaviour are then studied by simulation in Section 3 and 4, respectively. Micro-fabrication process is presented in Section 5. Calibration and validation of a real prototype will be developed in a further paper.

\section{Specifications and working principle}

\subsection{Sensor performance requirements}

As mentioned above, owing to their working principle, current commercial nanotribometers can only achieve a 2DOF force measurement leading sometimes to a non-negligible crosstalk which is not known and controlled.

Our new force sensor, that will be designed as a nanotribometer, should be able to simultaneously measure three-dimensional forces, torque and moments. The ability of torque and moments measurement will enable to access and decouple all friction components including rolling and spin motion. Each force measurement should achieve a linear and decoupled behaviour: hence, crosstalk has to be known and as low as possible. Moreover, the sensor should be able to host classical balls from different diameters met in tribological purpose. The normal (resp. tangential) force should have a maximum of $1 \mathrm{mN}$ $(100 \mu \mathrm{N})$ with a resolution of $1 \mu \mathrm{N}(0.1 \mu \mathrm{N})$ as currently applied in microgripping assembly.

\subsection{Working principle}

Keeping these performance requirements in mind, this sensor is composed of a central platform surrounded by a compliant table: four central beams and sixteen other beams form a table which enables to obtain higher displacements. The compliant table presents advantages compared with classical cross-beam sensors [21]: it leads us to obtain higher sensitivities reducing the number of gauges by increasing deformation and thus displacement ability. The ball that will rub against the studied surface is fixed to the central platform.

Figure 4 shows the multi-beam structure and the position of the eight strain gauges (named in the following G1,..,G8). The four sets of gauges are symmetrically distributed at the root of the four central beams.

To explain the sensing principle, a first focus on an isolated beam is done in Fig. 5 in order to illustrate the piezoresistivity-based operating mechanism. The piezoresistance effect is a phenomenon in which the electrical resistivity of a material changes due to an 
Figure 4 Architecture of the multi-axis force sensor.

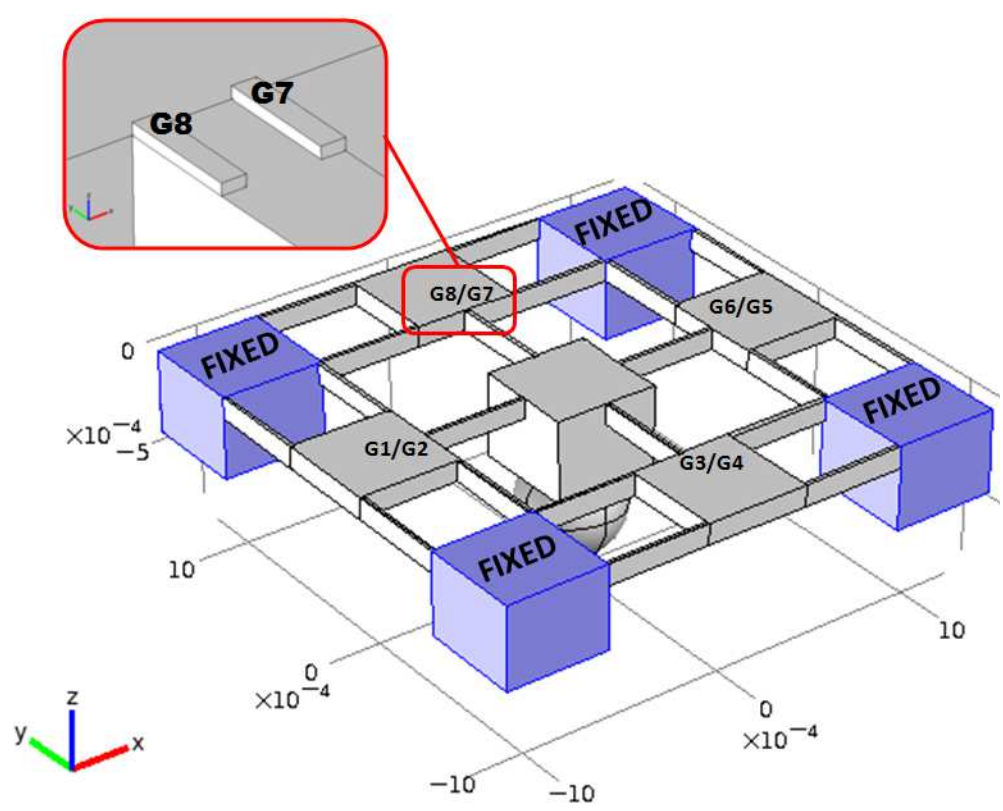

Figure 5 Working principle of the piezoresistance based measurement method (a: lateral force, b: normal force).

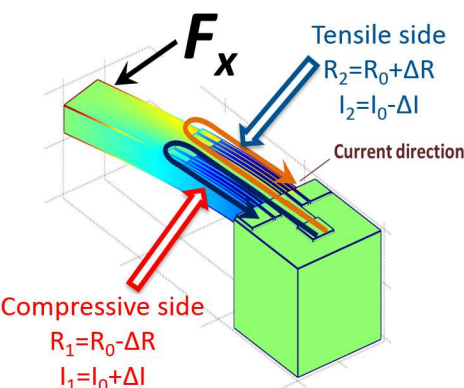

a

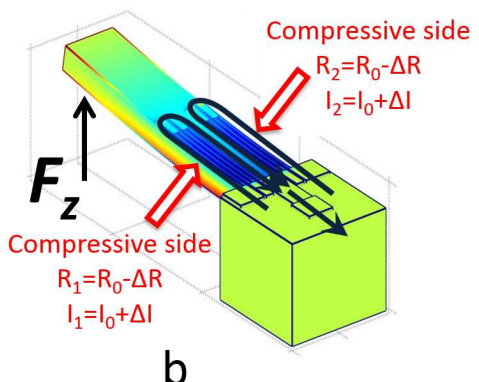

b

applied stress. In this paper, applied solicitations (forces and torque) are denoted Fx, Fy, Fz and $\mathrm{Cz}$, moments induced by forces are denoted $\mathrm{Mx}$ and $\mathrm{My}$, and measured components are denoted $\widehat{F x}$ and $\widehat{F y}$ (tangential forces corresponding to sliding), $\widehat{F z}$ (normal force), $\widehat{M x}$ and $\widehat{M y}$ (moments corresponding to rolling) and $\widehat{C z}$ (torque corresponding to spin motion).

As illustrated in Fig. 5, $I_{1}$ and $I_{2}$ are the current in the gauges 1 and 2, respectively, whereas $R_{1}$ and $R_{2}$ are the resistance of the strain gauges 1 and 2, respectively. So, the variations in the supply current (resp. resistance) of the strain-gauge 1 which is subjected to the compressive stress can be given by these equations:

$$
I_{1}=I_{0}+\Delta I
$$


Figure 6 Sensor under $\mathrm{Fx}, \mathrm{Fz}, \mathrm{Mx}$ and $\mathrm{Cz}$ constraint

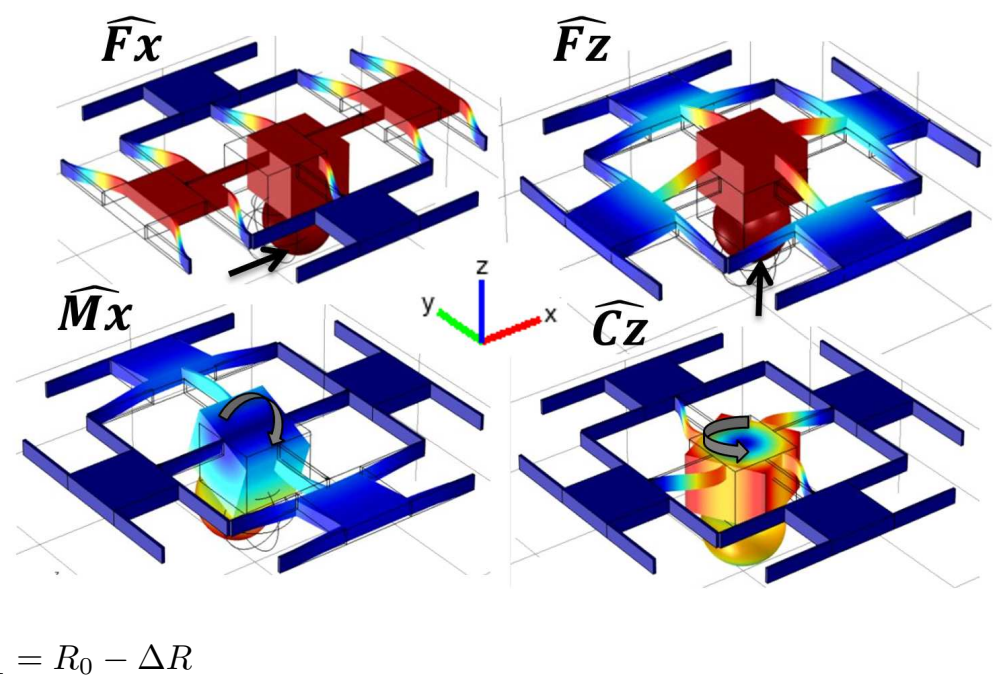

where, $I_{0}\left(R_{0}\right)$ is the initial current (resistance) value without any strain and $\Delta I(\Delta R)$ is the current (resistance) variation due to stress influence. The sign of $\Delta I$ and $\Delta R$ depends on the piezoresistor dopant type.

On the other hand, for the strain-gauge 2 under the tensile stress, current (resistance) variation is described by these equations:

$$
\begin{aligned}
& I_{2}=I_{0}-\Delta I \\
& R_{2}=R_{0}+\Delta R
\end{aligned}
$$

Hence, a tangential force induces different types of stresses on each side of the beam (see Fig. 5 a). Correspondingly, the two gauges have the opposite resistance variation. The difference of the resistance variation between the two strain-gauges can be then used to optimize the measurement sensitivity, as described by this relationship:

$$
R_{1}-R_{2}=R_{0}+\Delta R-\left(R_{0}-\Delta R\right)=2 \Delta R
$$

In a similar way, when a normal force is applied to the cantilever, both gauges are influenced by the same compressive or tensile stress (see Fig. 5b). Thus, the same resistance variation in these two gauges should be found (if each individual gauge has the same electrical behaviour). The following relationship is used to magnify the resistance variation:

$$
R_{1}+R_{2}=R_{0}+\Delta R+\left(R_{0}+\Delta R\right)=2 \Delta R+2 R_{0}
$$

By this way, the sum (resp. difference) of two gauges signals can be used to measure the normal (resp. lateral) forces.

Reasoning as so for the complete sensor, results obtained with each pair of gauges on the central beams are just summed, as summarized in Fig. 6and Tab. 1. For example, the tangential forces $\widehat{F y}, \widehat{F z}$ and moment $\widehat{M x}$ are evaluated using these formulas:

$$
\widehat{F y} \propto \Delta R_{G 1}-\Delta R_{G 2}-\Delta R_{G 5}+\Delta R_{G 6}=4 \Delta R_{\widehat{F y}}
$$


Table 1 Resistance variation in the gauges (smallest symbols represent a lower variation than others) for independent solicitations and their various combinations leading to possible crosstalks.

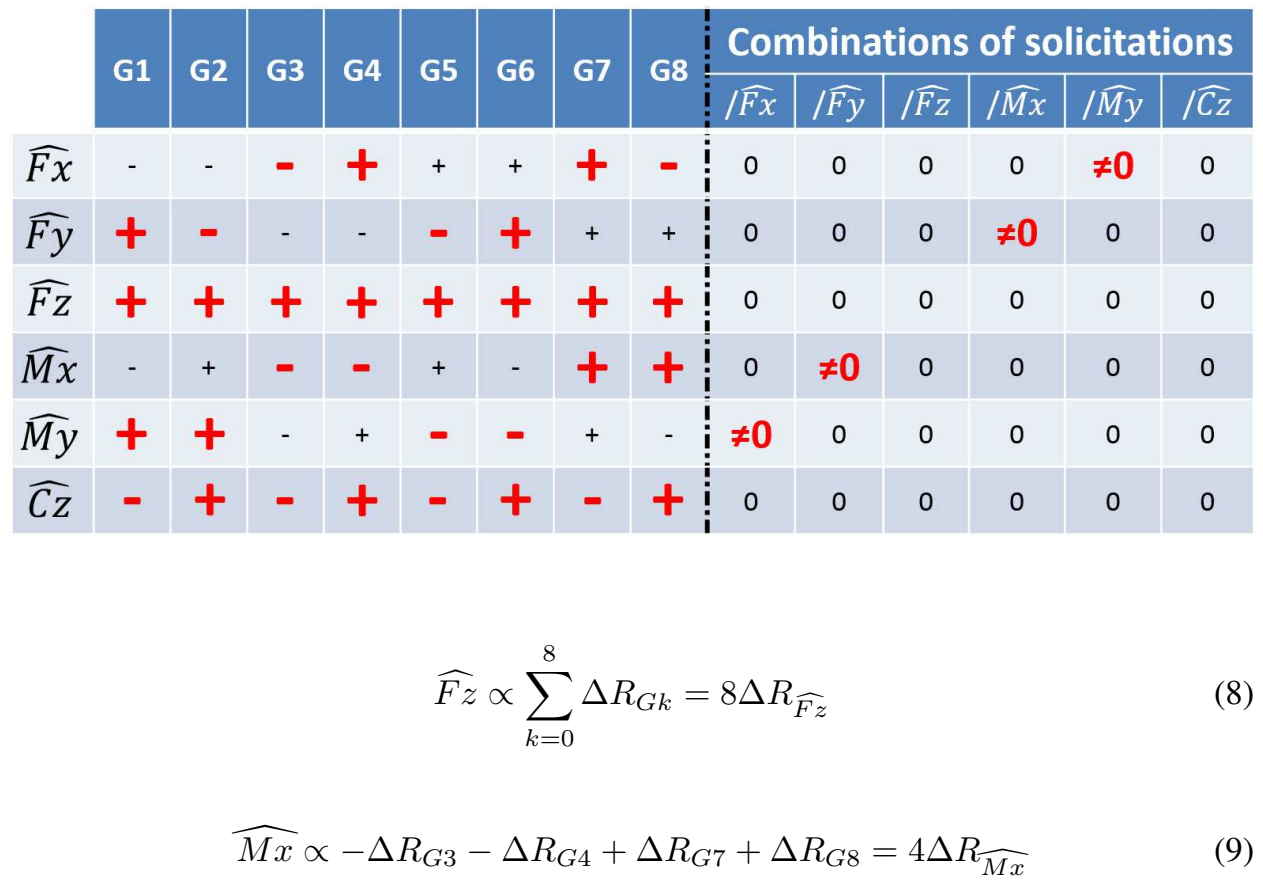

Moreover, smaller variations of resistances of gauges (denoted $\delta$ instead of $\Delta$ ) appear in some cases. For example, for a force $\widehat{F y}$ and a moment $\widehat{M x}$, these variations are:

$$
\begin{gathered}
4 \delta R_{\widehat{F y}}=-\Delta R_{G 3}-\Delta R_{G 4}+\Delta R_{G 7}+\Delta R_{G 8} \\
4 \delta R_{\widehat{M x}}=-\Delta R_{G 1}+\Delta R_{G 2}+\Delta R_{G 5}-\Delta R_{G 6}
\end{gathered}
$$

As shown in Tab. 1. which summarize the responses of the gauges submitted to independent solicitations and their various combinations leading to possible crosstalks, the compliant structure and the position of the eight piezoresistive gauges allow to completely decouple some components $(\widehat{F z}, \widehat{C z})$. However, a crosstalk between some other components for example $\widehat{F y}$ respect to $\widehat{M x}$ (which is the error on $\widehat{F y}$ measurement when there is a moment

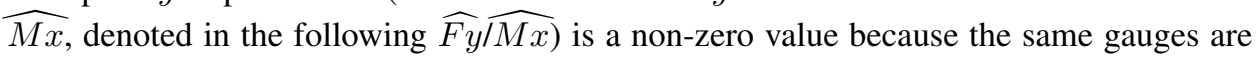
deformed in the same way for the two solicitations (see for example relationships 7 and 11. Indeed, for example, if the sensor is submitted to both tangential force Fy and moment $\mathrm{Mx}, \widehat{F y}$ is evaluated by:

$$
\widehat{F y} \propto 4 \Delta R_{\widehat{F y}}-4 \delta R_{\widehat{M x}} \neq 4 \Delta R_{\widehat{F y}}
$$

Nevertheless, simulation that is described in Section 4 will lead to study and find the best sensor geometry in order to limit these residual crosstalks (ie the ratio $\frac{\delta R_{\widehat{M x}}}{\Delta R_{\widehat{F y}}}$ for $\widehat{F y / M x}$ ). 
Figure 7 Young's modulus E and Poisson's ratio $\nu$ for wafer (100) [22].

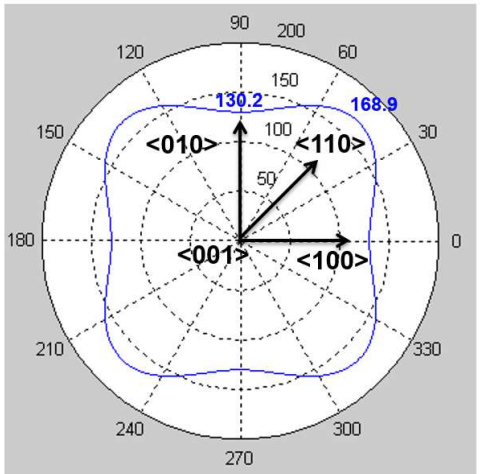

(100) E (GPa)

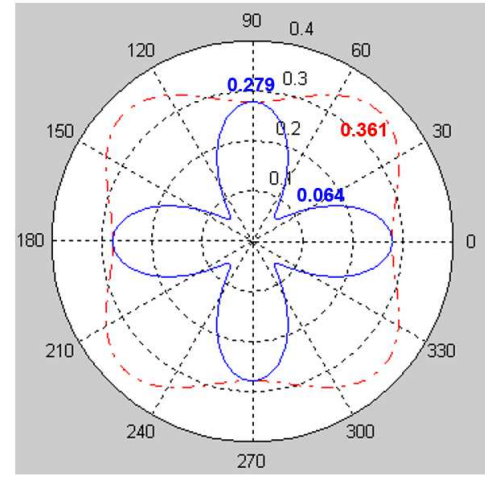

(100) $v_{/ /}$(solid line) $v_{\perp}$ (dotted line)

\section{Sensor design}

Knowing the sensor working principle, the optimization of the design has to be performed. The latter includes the choice of:

(i) the best crystallographic orientation of material constituting the compliant structure,

(ii) the type, size, orientation and position of the piezoresistive gauges.

Besides, a study of residual crosstalks using simulation will be carried out in order to find the best dimensions for the whole sensor to obtain optimal characteristics.

\subsection{Optimization of the mechanical structure orientation of the compliant structure}

The mechanical part is manufactured using Silicon micromachining fabrication technologies, to obtain a reasonably low stiffness structure. As Silicon is a highly anisotropic material, mechanical properties of the structure will depend on the crystallographic orientation of the chosen material. One criterion for the orientation choice is to have a low Young's modulus to improve the sensitivity of the sensor by reducing its stiffness [23], as shown in Fig. 7]and 8 from [22]. Although the two Young's modulus stay in the same order of magnitude by switching from Si (100) to Si (111), Young's modulus and Poisson's ratio are clearly independent of device crystallographic orientation for Silicon (111). Young's modulus is transversely isotropic at $168.9 \mathrm{GPa}$, regardless of the crystallographic orientation and Poisson's ratio has a constant value of $\nu_{/ /}=0.262$ for planes parallel to (111), and a constant value of $\nu_{\perp}=0.182$ for planes vertical to (111) [22]. Therefore, a sensor fabricated using (111) Silicon will be less sensitive to the compliant structure device orientation with respect to crystallographic orientations. Furthermore, since less restriction is imposed on mask designs with respect to crystallographic orientation, the design task becomes also much easier [22]. 
Figure 8 Young's modulus E and Poisson's ratio $\nu$ for wafer (111) [22].

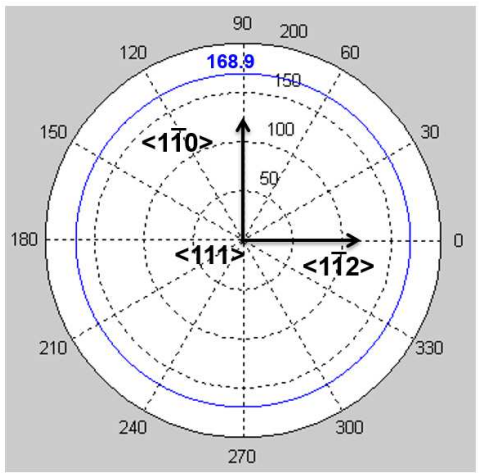

(111) E (GPa)

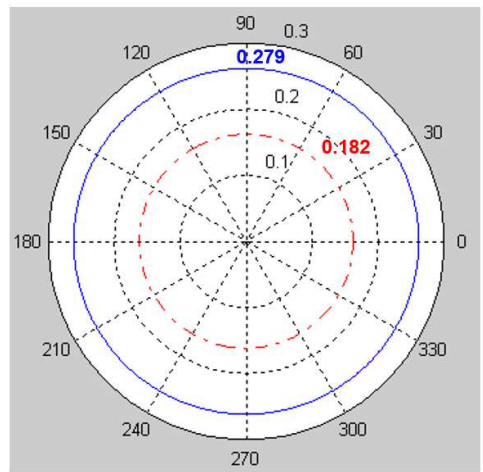

(111) $v_{/ /}$(solid line) $v_{\perp}$ (dotted line)

Figure 9 FEM model, dimensions of the meshed sensor and Stress distribution study: $\delta_{x}$ and $\delta_{y}$ plot the distance from edge side and embedding, respectively.

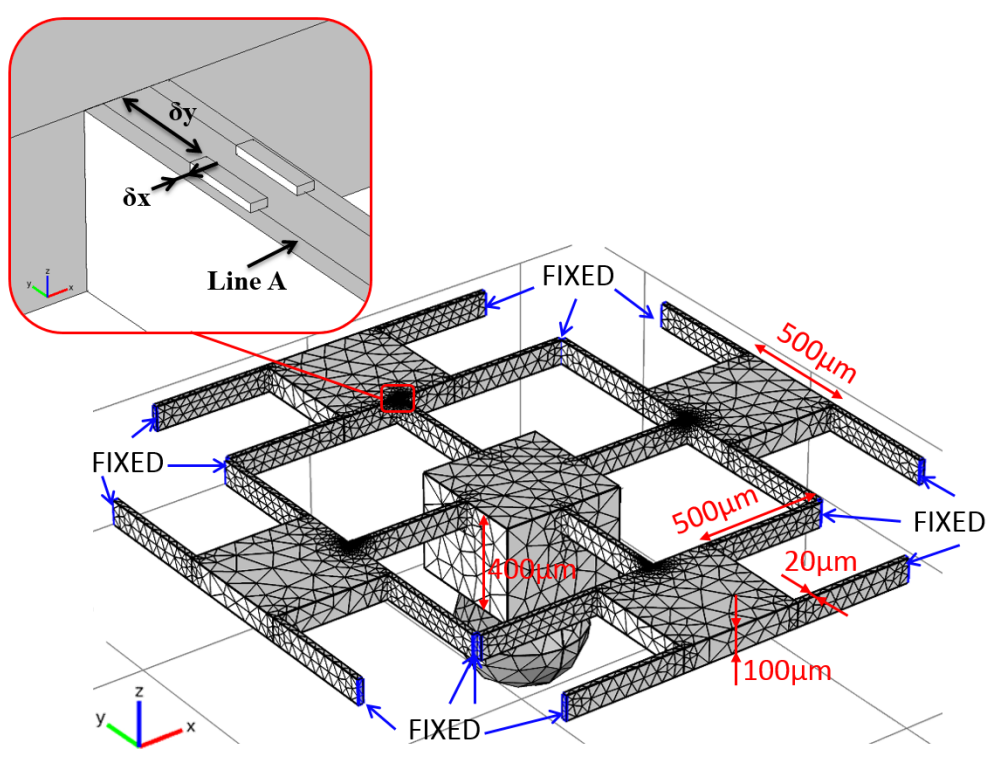


Figure 10 Stress distribution in the developed sensor (the component in solid line and higher than the others is $\sigma_{/ /}$).

\section{$\mathrm{Fx}=100 \mu \mathrm{N}$ with table (our sensor)}
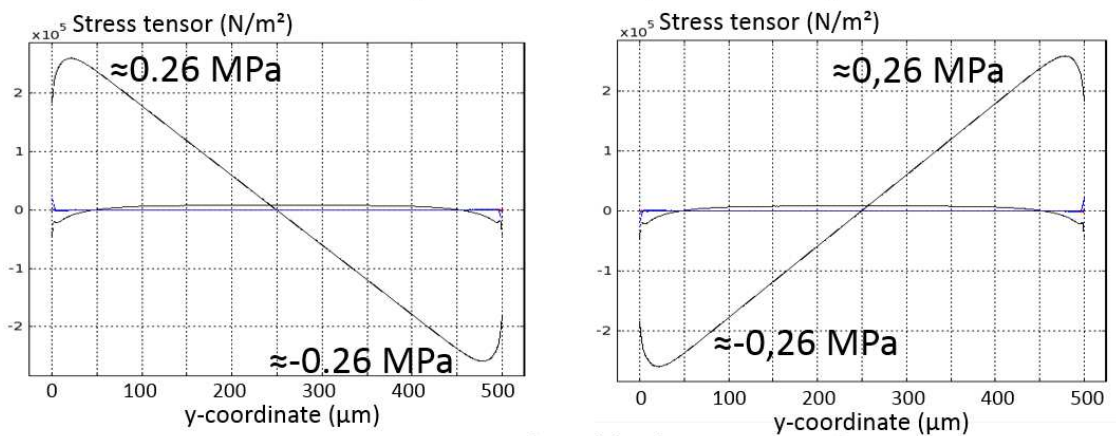

$\mathrm{Fz}=1000 \mu \mathrm{N}$ with table (our sensor)
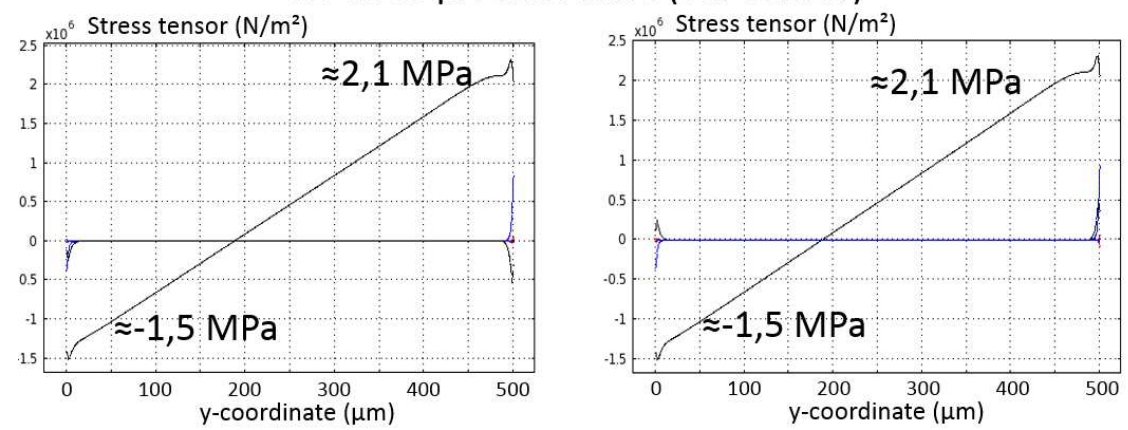

\subsection{Optimization of the piezoresistive gauges}

\subsubsection{Gauges position and size}

The stress distribution along different axis on the surface of the beam has been studied in order to determine the best position for the strain gauges. COMSOL Multiphysics Software 4.1 has been used to compare the results along the lines A defined on Fig. 9 for different $\delta_{x}$ and $\delta_{y}$ offset values. The "Structural Mechanics" module (linear elastic model) is used for this purpose and a tetrahedral meshing is realized (three nodes elements). To limit the calculation time, fixed parts are not modeled: the ends of the beams of the compliant table are directly fixed with a COMSOL boundary condition as shown in Fig. 9 Meshing is refined around the gauges to limit error induced by the discretization. A total of 26,240 elements is used (see Fig.99 and a powerful computing cluster is used (Mésocentre of calcul de Franche-Comté). Simulation results reveal that:

$\delta_{x}$ : The gauges have to be as far away from each other as possible $\left(\delta_{x}=0 \mathrm{~mm}\right)$ to obtain the higher stress differences between the two gauges from the same beam.

$\delta_{y}$ : Since the highest values of stress is observed at a distance of $\delta_{y}=20 \mu \mathrm{m}-$ as shown in Fig. 10 displaying stress repartition along the line A - the gauges should be placed a little far from the fixed support, to increase sensitivities. However, considering the difficulties induced by this shift in the current microfabrication process (electrical connections and ohmic contact achievements would be harder to realize), the gauges 
Figure 11 Notations of the dimensions of the gauges.

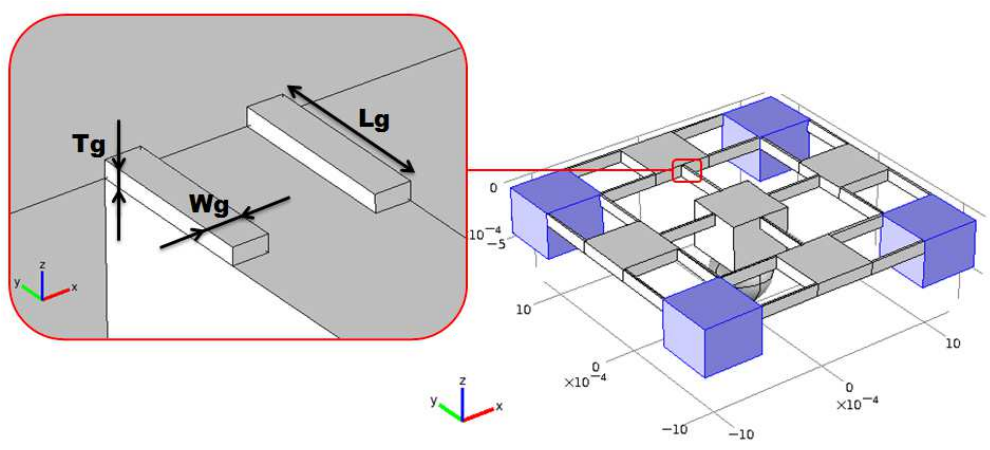

should be rather located at the clamped position $\left(\delta_{y}=0 \mathrm{~mm}\right)$. The loss of sensitivity value is evaluated around $6 \%$.

Piezoresistors' size has now to be optimized by choosing the dimensions displayed in Fig. 11. The gauges must be as small as possible in order to maximize sensitivity and not perturb structure deformation. According to the limitations of DRIE etching, the following gauges characteristics have been chosen:

- the length of the gauge $\mathrm{Lg}=50 \mu \mathrm{m}$;

- the height of the gauge $\mathrm{Tg}=1.5 \mu \mathrm{m}$;

- the width of the gauge $\mathrm{Wg}=3 \mu \mathrm{m}$;

- the resistivity of the gauge $\rho_{0}=0.01 \Omega . \mathrm{cm}$.

These dimensions induce a resistance of

$$
R_{0}=\frac{\rho_{0} L g}{S}=\frac{\rho_{0} L g}{W g T g}=1.1 \mathrm{k} \Omega
$$

when no stress is applied.

\subsubsection{Gauges type and orientation}

The piezoresistors lying on the surface of the sensing beams are very thin in comparison with the thickness of the beam. Therefore the piezoresistance effect of a piezoresistor can be expressed as :

$$
\frac{\Delta R}{R}=\pi_{/ /} \sigma_{/ /}+\pi_{\perp} \sigma_{\perp}
$$

where $\frac{\Delta R}{R}$ is the relative change of resistance, $\sigma_{/ /}$and $\sigma_{\perp}$ (resp. $\pi_{/ /}$and $\pi_{\perp}$ ) the two stress components (piezoresistive coefficients) parallel and orthogonal to the direction of the resistor, respectively. The piezoresistance coefficients depend both on the dopant and on the crystallographic orientation choice. Besides, for a given orientation, they can change significantly from one direction to another. Hence, sensor's sensitivity is then clearly 
Figure 12 Piezoresistive coefficients values for different wafer orientation (all values are in $10^{-12} \mathrm{~Pa}^{-1}$ ) from [24].

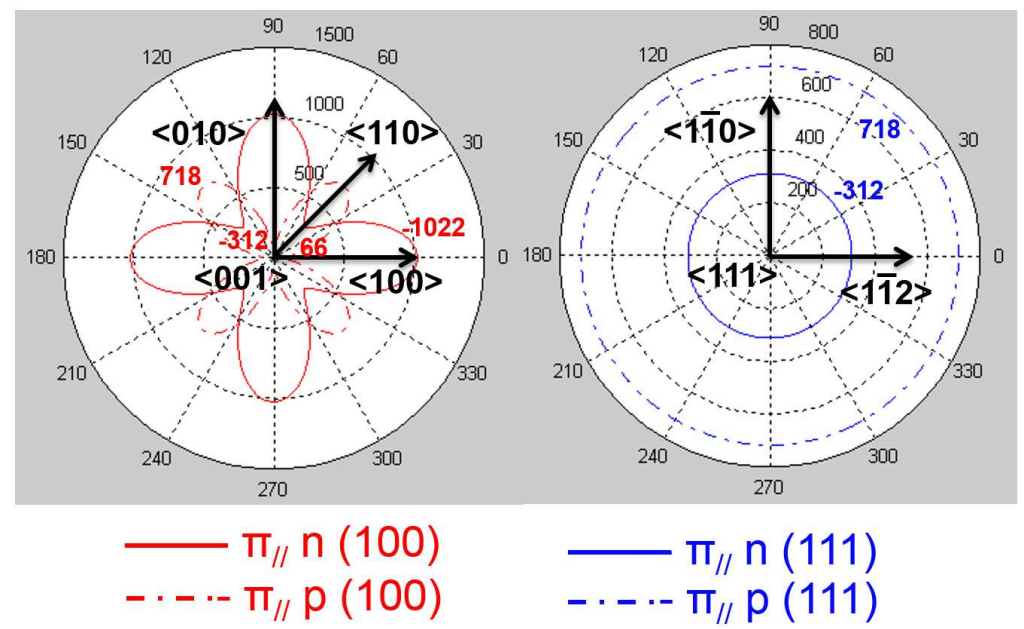

dependent on the crystallographic orientation. It is the main coercive criterion because crystallographic orientation is not determinant for DRIE manufacturing, in contrast to anisotropic humid etching [5].

Besides, stress analysis on the sensing structure surface (Fig. 10 in Section 3.2.1) shows that the component $\sigma_{/ /}$is much larger than other components of stress. For this reason, equation 14 can be simplify as :

$$
\frac{\Delta R}{R} \approx \pi_{/ /} \sigma_{/ /}
$$

The longitudinal gauge factor of the piezoresistance $\left(G F_{/ /}\right)$, which is the ratio between the relative variation of resistance and the gauge deformation, can then be expressed as :

$$
G F_{/ /} \approx \frac{\pi_{/ /} \sigma_{/ /}}{\epsilon_{/ /}} \Rightarrow G F_{/ /} \approx \pi_{/ /} E_{/ /}
$$

where $\epsilon_{/ /}$is the relative length change of the gauge and $E_{/ /}$is the Young's modulus in the direction parallel to the piezoresistor. Therefore, one criterion to select the crystallographic orientation is to make $\pi_{/ /} E_{/ /}$as large as possible. Figure 12 compares the value of $\pi_{/ /}$ for different orientations in $\mathrm{Si}$ (for the four different Si types: (111) and (100) doped $\mathrm{n}$ or p) [24]. And using results from Fig. 78 and 12, values of $G F_{/ /} \approx \pi_{/ /} E_{/ /}$can then be calculated and displayed in Fig. 13 Thus, for $\mathrm{Si}(111)$ the value of $G F_{/ /}$is clearly independent of the orientation of the gauge in contrast to $\mathrm{Si} \mathrm{(100).} \mathrm{It} \mathrm{could} \mathrm{be} \mathrm{an} \mathrm{advantage}$ if the gauges positions were not parallel or perpendicular, or even to avoid misalignment errors. Since higher gauge factor is obtained for a $\mathrm{Si}(100) \mathrm{n}$-doped and because all the gauges are oriented with the same crystallographic orientation in the our case, a n-doped (100) wafer with gauges that are oriented with the $<100>$ direction can be chosen to maximize the sensitivity. A gain of about $10 \%$ is expected. Note that, as $G F_{/ /}$is negative for n-doped $\mathrm{Si}, \Delta I$ and $\Delta R$ from Section 2.2 are negative too. 
Figure 13 Longitudinal gauge factor values for different wafer orientation.

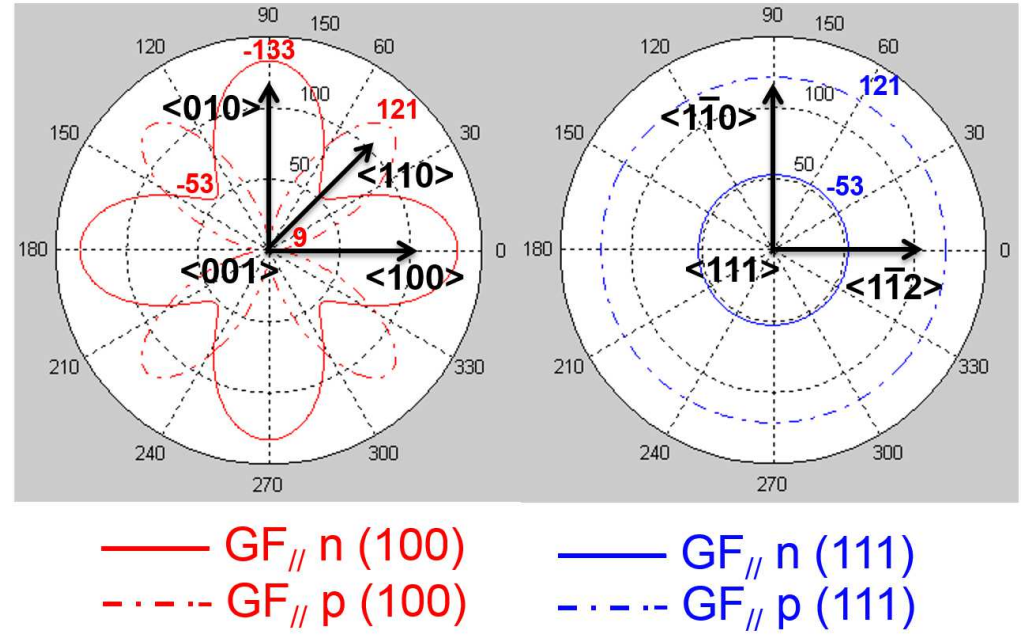

\section{Simulation of the sensor behaviour}

Simulations are now carried out in order to optimize the final design of the sensor. Expected performance and behaviour of the MEMS friction sensor are simulated using COMSOL multiphysics FEM simulation software. To take into account the anisotropy, the elasticity and piezoresistive matrices are used in the proper coordinate system. FEM model and meshing are the same as the ones described in Section 3.2.1. For piezoresistive effect, a co-simulation by using both the "Structural Mechanics" and "AC/DC" COMSOL modules is needed.

After meshing the geometrical structure and solving the differential equations of "Structural Mechanics" and "AC/DC" modules, the relative variation in resistance within a gauge is given by equation:

$$
\frac{\Delta R}{R}=\frac{\Delta I}{I}=\frac{\iint(J)-I_{0}}{I_{0}}
$$

in which $J$ is the current density norm inside the gauges determined in a plan orthogonal to the gauge direction (ie cross section of the gauge).

\subsection{Study of the crosstalks}

A Design of Experiments approach is used to determine the various crosstalks. Figure 14 shows the results for the structure deformation strengths, and the corresponding current variations in the gauges are related in Tab. 2. Values for gauges submitted to the same stress are averaged in order to erase small differences of current due to discretization errors. As expected, there is no crosstalk with normal force $\widehat{F z}$ or torque $\widehat{C z}$ (see Fig. 14 a and b and the corresponding lines in Tab. 2). Note that the ability of independently measuring a torque $\mathrm{Cz}$ with no error is important for our application because it enables to accurately characterize spin motion around the finger's apex, as represented in Fig. 3 c. However, a non-zero crosstalk is observed for $\widehat{F y} \widehat{M x}$ (and symmetrically for $\widehat{F x} / \widehat{M y}$ ) and $\widehat{M x} / \widehat{F y}$ 
Figure 14 Force and torque solicitations inducing a current variation in the gauges for different measurements: a normal force $\widehat{F z}($ a), a torque $\widehat{C z}$ (b), a tangential force $\widehat{F y}$ (c) and a moment $\widehat{M x}(\mathrm{~d})(\Delta I$ are positive values).

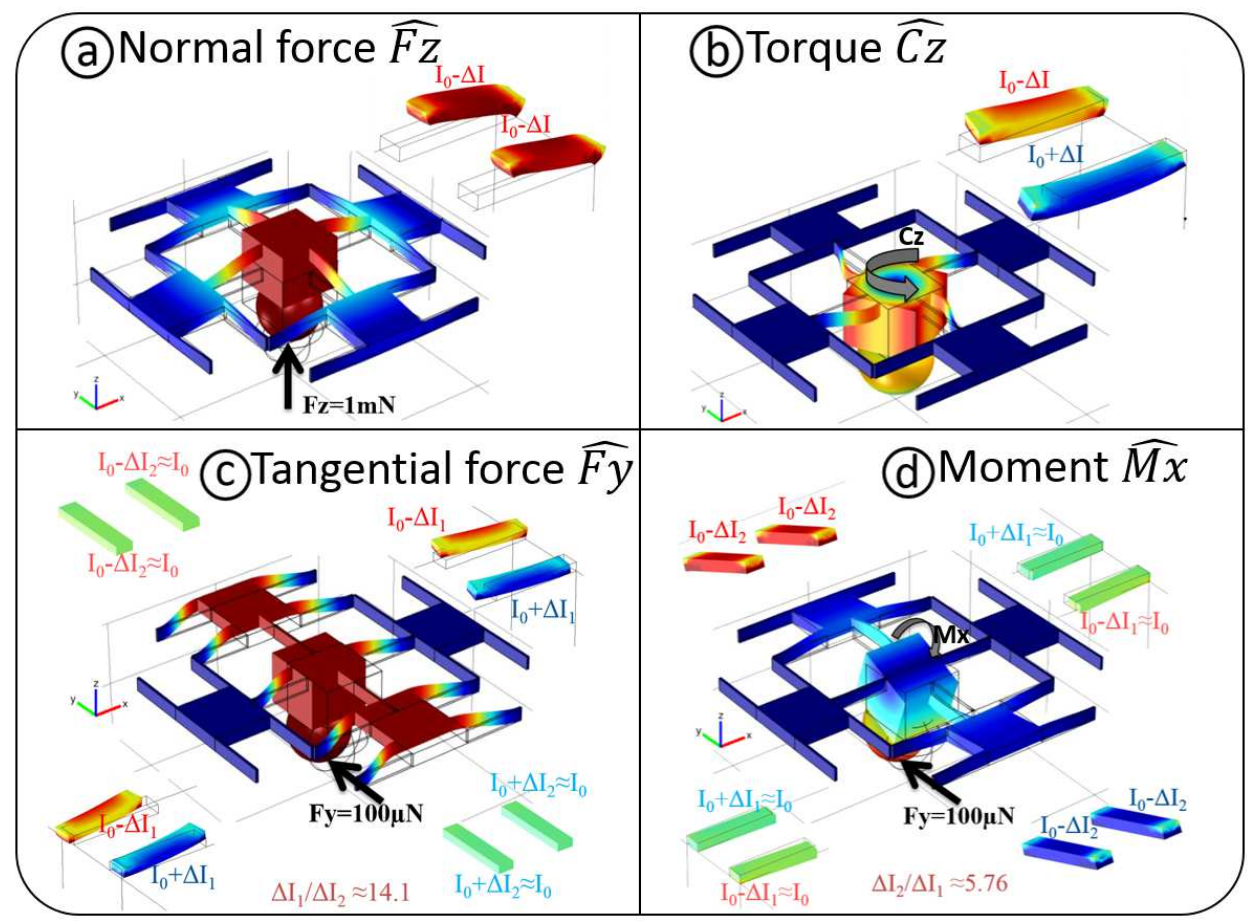

(and symmetrically for $\widehat{M y} / \widehat{F x}$ ) (notations being defined in the paragraph below Equation 11). Indeed, as shown in Fig. 14 c and d, if an unknown solicitation is applied on the sensor it will be, for example, impossible to extract the respective contribution of tangential force $\widehat{F y}$ and moment $\widehat{M x}$ from current variations of piezoresistive gauges. Referring to relation 12 and using the notations from Fig. $14 \mathrm{c}$ and d:

$$
\begin{aligned}
& \widehat{F y} \propto 4 \Delta I_{1}=4\left(\Delta I_{1 \widehat{F y}}-\Delta I_{1 \widehat{M x}}\right) \neq 4 \Delta I_{1 \widehat{F y}} \\
& \widehat{M x} \propto 4 \Delta I_{2}=4\left(\Delta I_{2 \widehat{M x}}+\Delta I_{2 \widehat{F y}}\right) \neq 4 \Delta I_{2 \widehat{M x}}
\end{aligned}
$$

Therefore an evaluation of the crosstalks levels is given by computing the ratios $\frac{\Delta I_{1 \widehat{M x}}}{\Delta I_{1 \widehat{F y}}}$ and $\frac{\Delta I_{2 \widehat{F y}}}{\Delta I_{2 \widehat{M x}}}$. Table 2 shows these residual errors: $7 \%$ for the evaluation of tangential force $\widehat{F y}$ and more than $17 \%$ for the moment $\widehat{M x}$. Although the latter is more important than the former, this difference has no real consequence on friction assessment in dexterous manipulation, as reported in Fig. 3 Indeed, in such framework, controlling the transition between static and dynamic friction is mandatory using the applied normal force knowing an estimation of the tangential force at the contact area. Hence, force measurements in 
Table 2 Current variation (nA) in the gauges for the six force components and corresponding non-zero crosstalks (values are given for $\mathrm{Fx}, \mathrm{y}=100 \mu \mathrm{N}$ and $\mathrm{Fz}=1000 \mu \mathrm{N}$ ).

\begin{tabular}{|c|c|c|c|c|c|c|c|c|c|}
\hline & G1 & G2 & G3 & $\mathbf{G 4}$ & G5 & G6 & G7 & G8 & $\begin{array}{l}\text { Non-zero } \\
\text { crosstalks }\end{array}$ \\
\hline$\widehat{F x}$ & 12.23 & 12.23 & 172.17 & 72.17 & -12.23 & -12.23 & -172.17 & 172.17 & $/ \widehat{M y}: 7.18 \%$ \\
\hline$\widehat{F y}$ & -172.17 & 72.17 & 12.23 & 12.23 & 172.17 & -172.17 & -12.23 & -12.23 & $/ \widehat{M x}$ \\
\hline$\widehat{F Z}$ & -782.20 & -782.20 & -782.20 & -782.20 & -782.20 & -782.20 & -782.20 & -782.20 & \\
\hline$\widehat{M x}$ & 12.36 & -12.36 & 71.15 & 71.15 & -12.36 & 12.36 & -71.15 & -71.15 & /Fy: $17.18 \%$ \\
\hline$\widehat{M y}$ & -71.15 & - & 36 & 1.00 & 71.15 & s. & 12.00 & 12.36 & $\widehat{F x}: 17.18 \%$ \\
\hline$\widehat{C Z}$ & 320.08 & -320.08 & 320.08 & -320.08 & 320.08 & -320.08 & 320.08 & -320.08 & \\
\hline
\end{tabular}

sliding mode must be accurate, even in presence of residual crosstalks induced by any rolling process generated by a geometric rotation of the central platform (ie, presence of moments). On the contrary, accurately measurement of a moment $\widehat{M x}$ or $\widehat{M y}$ induced by a rolling at the contact area appears less critical because, in dexterous framework, the crucial information is just to make sure to keep the contact during the movement. Nevertheless, a solution for reducing the crosstalks is to modify the sensor design by placing the ball on the upper side of the sensor and reducing the height of the central platform. By this way, the distance $T_{s}$ between the support plane and the flat sample strongly decreases, as shown in Fig. 15. The rotation components will be less important so that the error on the friction forces will be reduced. To perform that, a hole is designed in the central platform and the micro-ball is glued in it. The diameter of the hole can be adapted to host balls from different sizes without changing the distance between the sample and the central platform. With this design modification, the sensor will work upside-down with the gauges placed in front of the studied sample (see Fig. 15).

\subsection{Optimization of the sensor design}

In addition to the above crosstalk study, there are many geometrical characteristics described in Fig. 16 that also influence the sensor properties:

- the length $L$ of the beams;

- the thickness $T$ of the beams;

- the width $W$ of the beams;

- the distance $T_{s}$ between the sample and the central platform;

- the length $L_{p}$ of the central platform;

- the thickness $T_{p}$ of the central platform.

Several criteria are selected to check their influence out: (i) the performance analysis of decoupling, (ii) the resonant frequency, (iii) the sensitivities, (iv) the response time and (v) the maximum forces that can be applied before the sensor rupture. All the simulations results are summarized in Tab. 3. The upper line of the table corresponds to the variation 
Figure 15 Modification of the sensor design in order to decrease crosstalks: the sensor works upside-down with the gauges at the bottom.
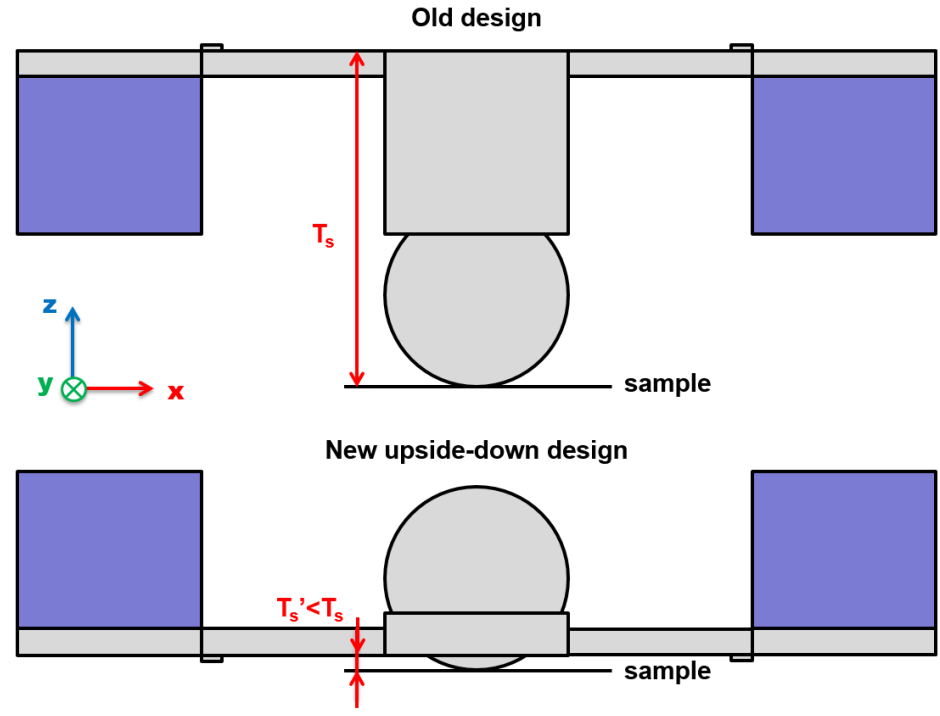

Figure 16 Notations of the geometrical parameters of the sensor (with the ball on the upper side of the platform).

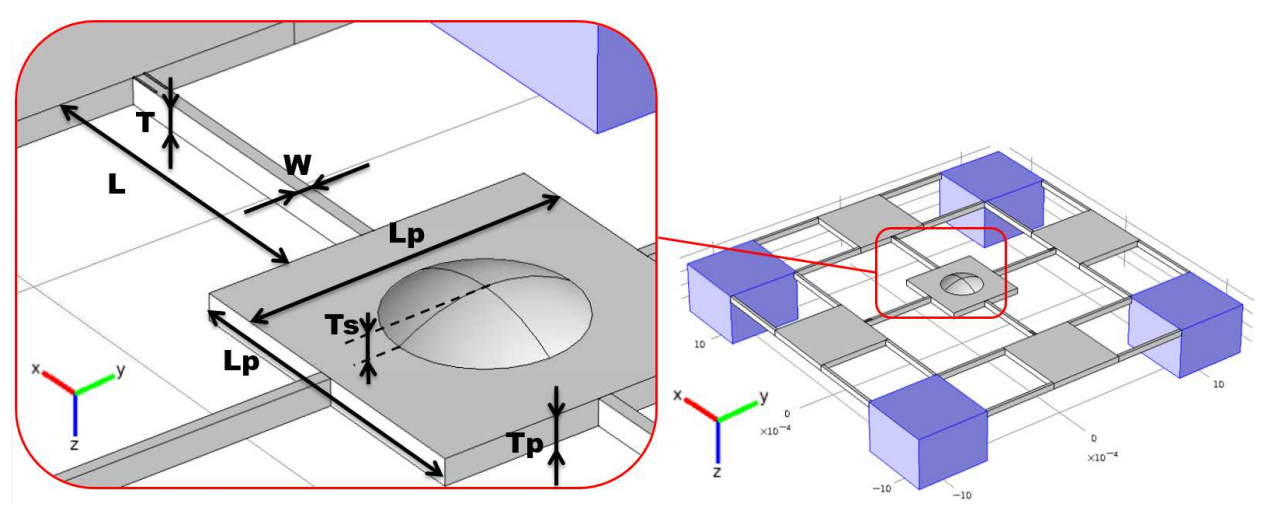


Table 3 Study of the influence of the geometrical parameters (+ (++,-) means that the variation of the parameter have a positive (very positive, negative) influence on the considered sensor property and 0 $(0 /-)$ means that the variation of the parameter have no influence (a negligible negative influence, respectively) on the sensor property, under bracket expressions are the eventual proportionality relationships between geometrical parameters and characteristics).

\begin{tabular}{|c|c|c|c|c|c|c|c|}
\hline \multicolumn{2}{|c|}{$\begin{array}{l}\text { Variation of the geometrical } \\
\text { Characteristics parameter } \\
\text { and selection criteria }\end{array}$} & $\mathbf{y} \mathrm{T}$ & $\lambda \mathrm{L}$ & $\mathbf{y} W$ & Y Ts & $\lambda \mathrm{Lp}$ & Y Tp \\
\hline Resonance frequency & Maximize & $0 /-$ & - & - & + & 0 & + \\
\hline Sensitivity $\widehat{F x}, \widehat{F y}$ & Maximize & $+(\propto 1 / T)$ & $+(\propto L)$ & + & $0 /-$ & + & 0 \\
\hline Sensitivity $\widehat{F Z}$ & Maximize & $++\left(\propto 1 / T^{2}\right)$ & + & + & $0 /-$ & + & 0 \\
\hline Stiffness Kx,Ky & Minimize & $+(\propto T)$ & $++\left(\propto 1 / L^{3}\right)$ & $++\left(\propto W^{3}\right)$ & $0 /-$ & 0 & 0 \\
\hline Stiffness Kz & Minimize & $++\left(\propto T^{3}\right)$ & $++\left(\propto 1 / L^{3}\right)$ & $+(\propto W)$ & $0 /-$ & + & 0 \\
\hline Crosstalk $\widehat{F x, y} / \widehat{M y, x}$ & Minimize & 0 & + & + & + & + & + \\
\hline Crosstalk $\overline{M x, y} / \overline{F y, x}$ & Minimize & 0 & $0 /-$ & $0 /-$ & - & + & 0 \\
\hline Response time & Minimize & - & - & - & + & - & + \\
\hline $\begin{array}{l}\text { Maximal force before } \\
\text { rupture }\end{array}$ & Maximize & - & - & - & 0 & 0 & 0 \\
\hline
\end{tabular}

of the different geometrical parameters (augmentation $\nearrow$ or reduction $\searrow$ ) and in the first two columns there are the characteristics and the corresponding criteria to be maximized or minimized, that is:

- sensitivities have to be as high as possible;

- the resonant frequency has to be high enough to avoid environmental perturbations due to vibrations during the measurement (higher than $10 \mathrm{kHz}$ ) [25, 26];

- a low stiffness is needed in order to obtain big enough displacements;

- crosstalk between forces and moments measurement needs to be negligible (maximum 1 or $2 \%$ ) to respect the sensor requirements;

- a short response time is needed to efficiently measure the various components during friction process;

- and the maximum force before rupture has to be as high as possible, in order to maximize the force range.

Another last criterion which is unmeasurable is the ability to easily fabricate and manipulate this small sensor without breaking it.

Given Tab. 3, the best dimensions are selected making a trade-off between the good and the bad influence of parameters on the different characteristics, knowing that crosstalk, sensitivities and response time are the main important choice criteria. Consequently, the beam height $\mathrm{T}$ and the beam width $\mathrm{W}$ are reduced whereas the beam length $\mathrm{L}$ is increased as much as possible to obtain high sensitivities. But, as these variations have simultaneously bad effects on several other characteristics, their values are chosen in order to not increase 
Figure 17 Geometrical parameters of the sensor (all the dimensions are in $\mu \mathrm{m}$ ).

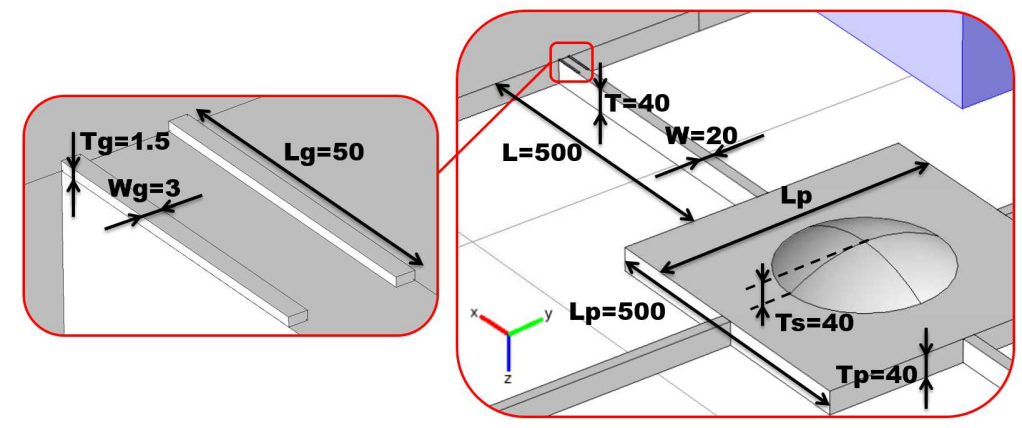

too much the crosstalk $\widehat{M x} / \widehat{F y}$ and the response time. Moreover, both the distance between the sample and the central platform $T_{s}$ and the thickness of the central platform $T_{p}$ are reduced to obtain a higher resonant frequency and a shorter response time. The smallest ball size usually used to perform tribological tests in the lab is retained for $T_{s}(40 \mu \mathrm{m})$. Indeed, as mentioned above, reducing the distance from the sample has no bad influence on other important parameters (sensitivities, crosstalk, etc.). Hence, the same thickness for central platform and beams is selected to simplify the fabrication process. And a small enough value of $L_{p}$ is then chosen in order to both minimize the response time and reduce the sensor size.

Finally, the optimal geometrical parameters obtained from simulation results are reported in Fig. 17

\subsection{Characteristics of the final sensor}

With the optimized dimensions, the sensor displays the expected properties compiled in Tab. 4 Crosstalks values of the sensor are reported in Tab. 5 . Characteristics have been improved with respect to the first design (see Tab. 2 from Section 4.1). Although the residual errors on moment $\widehat{M x}$ have slightly increased - from $17.2 \%$ to $19.1 \%$ - without any real consequence on our application, as mentioned earlier (see Section 4.1), those have significantly decreased - from $7.2 \%$ to $0.71 \%$ - on tangential force $\widehat{F y}$. It means that measurement of a tangential force induced by a torque can be accurately decoupled with a great benefit. Of course, these properties could slightly change on the real sensor because of the uncertainties due to micro-manufacturing process. However, the best was made in order to have low crosstalks, good sensitivities and a resonant frequency high enough to avoid perturbations due to environmental noise (acoustic and seismic vibrations [25, 26]) during the measurement. Note that, the actual design of the sensor has been determined by simulations to respond to our experimental nanotribological requirements (micro newton force range measurement with no restriction on the structure size). However, this optimization process can be clearly used to design a nanosensor displaying other kinds of requirements. Indeed, as in our FEM model all the dimensions and material properties can be easily parametrized, our optimization process can be applied to identify the optimal dimensions and so for the purpose of scalability. The only limit of our FEM model is to stay in the field of the classical mechanics. 
Table 4 Characteristics of the sensor (simulation results).

\begin{tabular}{|c|c|c|c|c|c|c|}
\hline \multicolumn{4}{|c|}{ Sensitivities } & $\begin{array}{l}\text { Resonant } \\
\text { frequency }\end{array}$ & \multicolumn{2}{|c|}{ Stiffness } \\
\hline $\begin{array}{c}\mathbf{S}_{\widehat{F} \widehat{x, y}} \\
(\mathrm{nA} / \mu \mathrm{N}\end{array}$ & $\begin{array}{c}\mathbf{S}_{\hat{F Z}} \\
(n A / \mu N\end{array}$ & $\begin{array}{c}\mathbf{S}_{\mathrm{M} \widehat{x}, \mathbf{y}} \\
(\mathrm{nA} / \mu \mathrm{rad})\end{array}$ & $\begin{array}{c}\mathbf{S}_{\widehat{C} \mathbf{z}} \\
(\mathrm{nA} / \mu \mathrm{rad})\end{array}$ & $\begin{array}{l}F_{\text {res }} \\
(\mathrm{kHz})\end{array}$ & $\begin{array}{c}\mathbf{K}_{\mathrm{x}, \mathrm{y}} \\
(\mathrm{kN} / \mathrm{m})\end{array}$ & $\begin{array}{c}\mathbf{K}_{\mathbf{z}} \\
(\mathrm{kN} / \mathrm{m})\end{array}$ \\
\hline 17.3 & 43.2 & 12.9 & 229 & 36.6 & 4.10 & 2.31 \\
\hline \multicolumn{3}{|c|}{$\begin{array}{l}\text { Maximal forces / } \\
\text { displacements before rupture }\end{array}$} & \multicolumn{2}{|c|}{ Crosstalks } & \multicolumn{2}{|c|}{ Response times } \\
\hline \multicolumn{2}{|c|}{$\begin{array}{c}\mathbf{F}_{x, y \max }(\mathrm{mN}) / \\
\mathbf{D}_{x, y \max }(\mu \mathrm{m})\end{array}$} & $\begin{array}{l}\mathbf{F}_{\text {zmax }}(\mathrm{mN}) \\
\mathbf{D}_{\text {zmax }}(\mu \mathrm{m})\end{array}$ & $\begin{array}{c}\widehat{F x, y} / \widehat{M y, x} \\
(\%)\end{array}$ & $\begin{array}{c}\bar{x} \overline{x, y} / F y, x \\
(\%)\end{array}$ & $\begin{array}{ll}\mathbf{x} & \tau_{\mathrm{F} \widehat{x}, \mathbf{y}} \\
& (\mathrm{ms})\end{array}$ & $\begin{array}{c}\tau_{\widehat{F}} \\
(\mathrm{~ms})\end{array}$ \\
\hline \multicolumn{2}{|c|}{$\begin{array}{l}33.5 \\
/ 8.16\end{array}$} & $\begin{array}{l}22.9 \\
/ 9.94\end{array}$ & 0.713 & 19 & 1.11 & 1.40 \\
\hline
\end{tabular}

Table 5 Current variation (nA) in the gauges for the six measured components and corresponding non-zero crosstalks (values are given for $\mathrm{Fx}, \mathrm{y}=100 \mu \mathrm{N}$ and $\mathrm{Fz}=1000 \mu \mathrm{N}$ ).

\begin{tabular}{|c|c|c|c|c|c|c|c|c|c|}
\hline & G1 & G2 & G3 & G4 & G5 & G6 & G7 & G8 & $\begin{array}{l}\text { Non-zero } \\
\text { crosstalks }\end{array}$ \\
\hline$\widehat{F x}$ & 8.59 & 8.59 & 431.33 & -431.33 & -8.59 & -8.59 & -431.33 & 431.33 & $/ \widehat{M y}: 0.713 \%$ \\
\hline$\widehat{F y}$ & -431.33 & 431.33 & 8.59 & 8.59 & 431.33 & -431.33 & -8.59 & -8.59 & $/ \widehat{M x}: 0.713 \%$ \\
\hline$\widehat{F Z}$ & -5394.11 & -5394.11 & -5394.11 & -5394.11 & -5394.11 & -5394.11 & -5394.11 & -5394.11 & \\
\hline$\widehat{M x}$ & 3.08 & -3.08 & 44.90 & 44.90 & -3.08 & 3.08 & -44.90 & -44.90 & /Fy: $19.13 \%$ \\
\hline$\widehat{M y}$ & -44.90 & -44.90 & 3.08 & -3.08 & 44.90 & 44.90 & -3.08 & 3.08 & $\widehat{F x}: 19.13 \%$ \\
\hline$\widehat{C Z}$ & 810.65 & -810.65 & 810.65 & -810.65 & 810.65 & -810.65 & 810.65 & -810.65 & \\
\hline
\end{tabular}


Figure 18 Flow chart: ( 1 a) SOI wafer with 5 layers ( 1 b) lithography and DRIE etching upper side of the device layer ( 2 a) thermic oxidation ( 2 b) lithography and RIE etching for opening the ohmic contact areas $(2 \mathrm{c}) \mathrm{Cr} / \mathrm{Pt}$ deposition, lithography, wet etching and diffusion heating to realize the ohmic contacts (3) $\mathrm{Cr} / \mathrm{Pt}$ deposition, lithography and wet etching to obtain electrical connection electrodes (4 a) lithography and DRIE etching down side of the handle layer (4 b) lithography and DRIE etching upper side of the handle layer.

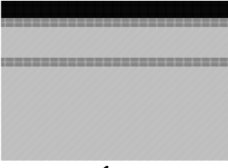

1 a

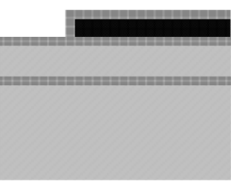

$2 \mathrm{a}$

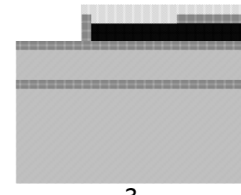

3

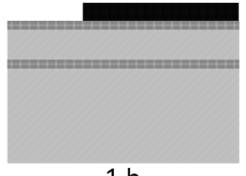

$1 \mathrm{~b}$

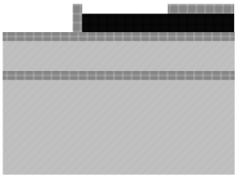

$2 b$

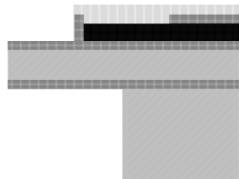

4 a

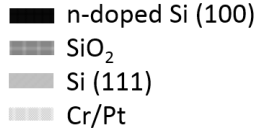

n-doped Si (100)

$\mathrm{SiO}_{2}$

Si (111)

$\mathrm{Cr} / \mathrm{Pt}$

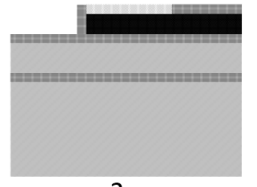

$2 \mathrm{c}$

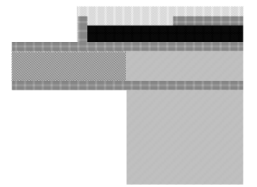

$4 \mathrm{~b}$

\section{Fabrication process}

Our sensor will be manufactured on a n type silicon SOI wafer. In order to obtain a compliant structure as perfect as possible a five layers SOI wafer is used. Tests are at the moment being performed to optimize ohmic contacts achievement for gauges realization [27]. Then once optimal metal thicknesses, time and temperature for the annealing proceeding will be determined, sensor micromachining will be launched. Fabrication process will typically consist of the four stages illustrated in Figure 18

1. Piezo-resistors achievement:

- The piezoresistive gauges are defined using deep reactive ion etching of silicon.

2. Ohmic contact achievement:

- The thermal oxidation treatment of piezoresistive gauge is carried out as an electrical insulator layer.

- To open the contact windows, the reactive ion etching is used to etch silicon dioxide.

- A Chrome/Platinum layer is deposited and patterned by the evaporation deposition and lift-off methods.

- The annealing proceeding is needed to have the ohmic contact between Platinum and silicon layers.

3. Connection circuit achievements :

- A Platinum layer is deposited by the sputtering method. 
- The Platinum layer is patterned by wet etching method to achieve the electrical connection.

4. Sensor structure achievements:

- To create the beams, the back side of bulk is etched by deep ion reactive etching.

- To realize the sensor structure, the front side of bulk is etched by deep ion reactive etching.

\section{Conclusion}

A new multi-axis force sensor which is intended to measure frictional components involved in dexterous microgripping was developed. The design of the sensor based on a compliant table and all its geometrical properties were studied in order to optimize sensitivity and resolution and to minimize drawbacks. The first simulation results are promising: this sensor will be able to measure independently the normal and friction forces (crosstalk inferior to $1 \%$ ) with a good sensitivity. Tests are at the moment being performed in order to optimize ohmic contacts achievement for gauges realization and make a final choice between $\mathrm{n}$ and $\mathrm{p}$-type Si. Then once optimal metal thicknesses, time and temperature for the annealing proceeding will be determined, sensor micromachining will be launched and then experimental results will be compared with simulation ones. A big challenge to overcome will be the development of a process to be able to efficiently calibrate the sensor.

\section{Acknowledgements}

This work has been supported by the Labex ACTION project (contract "ANR-11-LABX-01$01 ")$. Calculations have been performed using the computing resources from the Mésocentre of Franche-Comté. This work has been supported by the French RENATECH network and its FEMTO-ST technological facility.

\section{References}

[1] Pierre Lambert. Capillary forces in microassembly: modeling, simulation, experiments, and case study. Springer, 2007.

[2] Karl Goser, Jan Dienstuhl, et al. Nanoelectronics and nanosystems: from transistors to molecular and quantum devices. Springer, 2004.

[3] Enrico Gnecco and Ernst Meyer. Fundamentals of friction and wear. Springer, 2007.

[4] T Pradeep. NANO: The Essentials: Understanding Nanoscience and Nanotechnology. Mc Graw Hill, 2007.

[5] Tai-Ran Hsu. MEMS \& Microsystems: Design, Manufacture, and Nanoscale Engineering. John Wiley \& Sons, 2008. 
[6] Joël Agnus, Nicolas Chaillet, Cédric Clévy, Sounkalo Dembélé, Michaël Gauthier, Yassine Haddab, Guillaume Laurent, Philippe Lutz, Nadine Piat, Kanty Rabenorosoa, et al. Robotic microassembly and micromanipulation at FEMTO-ST. Journal of Micro-Bio Robotics, 8(2):91-106, 2013.

[7] David Hériban and Michaël Gauthier. Robotic micro-assembly of microparts using a piezogripper. In IEEE/RSJ International Conference on Intelligent Robots and Systems, 2008. IROS 2008., pages 4042-4047. IEEE, 2008.

[8] Sylwester Bargiel, Kanty Rabenorosoa, Cédric Clevy, Christophe Gorecki, and Philippe Lutz. Towards micro-assembly of hybrid MOEMS components on a reconfigurable silicon free-space micro-optical bench. Journal of Micromechanics and Microengineering, 20(4):045012, 2010.

[9] Kanty Rabenorosoa, Cédric Clévy, Philippe Lutz, Sylwester Bargiel, and Christophe Gorecki. A micro-assembly station used for 3D reconfigurable hybrid moems assembly. In Assembly and Manufacturing, 2009. ISAM 2009. IEEE International Symposium on, pages 95-100. IEEE, 2009.

[10] Satish Achanta and Jean-Pierre Celis. Nanotribology of mems/nems. In Enrico Gnecco and Ernst Meyer, editors, Fundamentals of Friction and Wear, NanoScience and Technology, pages 521-547. Springer Berlin Heidelberg, 2007.

[11] Sandeep Krishnan and Laxman Saggere. A multi-fingered micromechanism for coordinated micro/nano manipulation. Journal of Micromechanics and Microengineering, 17(3):576, 2007.

[12] Quan Zhou, Petteri Korhonen, Jukka Laitinen, and Sami Sjovall. Automatic dextrous microhandling based on a 6-DOF microgripper. Journal of Micromechatronics, 3(34):3-4, 2006.

[13] Philippe Stempflé, Aurélien Besnard, Nicolas Martin, Anne Domatti, and Jamal Takadoum. Accurate control of friction with nanosculptured thin coatings: Application to gripping in microscale assembly. Tribology International, 59:67-78, 2013.

[14] OM Braun and AG Naumovets. Nanotribology: Microscopic mechanisms of friction. Surface Science Reports, 60(6):79-158, 2006.

[15] Izabela Szlufarska, Michael Chandross, and Robert W Carpick. Recent advances in single-asperity nanotribology. Journal of Physics D: Applied Physics, 41(12):123001, 2008.

[16] Anne Domatti, Philippe Stempflé, Pascal Carrière, and Jamal Takadoum. Multiasperity nanotribology of self-assembled monolayers grafted on silicon wafers displaying various crystallographic orientations and nanostructures. Tribology Letters, 51(2):207-218, 2013.

[17] Satish Achanta, Dirk Drees, and Jean-Pierre Celis. Investigation of friction on hard homogeneous coatings during reciprocating tests at micro-newton normal forces. Wear, 263(7):1390-1396, 2007. 
[18] T Zijlstra, JA Heimberg, E Van der Drift, D Glastra van Loon, M Dienwiebel, LEM De Groot, and JWM Frenken. Fabrication of a novel scanning probe device for quantitative nanotribology. Sensors and Actuators A: Physical, 84(1):18-24, 2000.

[19] W.L. Jin and C.D. Mote. Development and calibration of a sub-millimeter threecomponent force sensor. Sensors and Actuators A: Physical, 65(1):89-94, 1998.

[20] P Estevez, J M Bank, M Porta, J Wei, P M Sarro, M Tichem, and U Staufer. 6 DOF force and torque sensor for micro-manipulation applications. Sensors and Actuators A: Physical, 186:86-93, 2012.

[21] Dzung Viet Dao, Toshiyuki Toriyama, John Wells, and Susumu Sugiyama. Silicon Piezoresistive Six-Degree of Freedom Force-Moment Micro Sensor. 2004.

[22] Jongpal Kim, D Cho, and Richard S Muller. Why is (111) silicon a better mechanical material for MEMS ? Proceedings of the 11th International Conference on Solid-state Sensors and Actuators (Transducers 01), Munich, Germany, pages 662-665, 2001.

[23] A. Jordan and S. Büttgenbach. Micromechanical force sensors based on SU-8 resist. Microsystem Technologies, 18(7-8):1095-1101, 2012.

[24] Yoko Kanda. A Graphical Representation of the Piezoresistance Coefficients in Silicon. IEEE Transaction on electron devices, ED-29(1):64-70, january 1982.

[25] Mokrane Boudaoud, Yassine Haddab, Yann Le Gorrec, and Philippe Lutz. Effects of environmental noise on the accuracy of millimeter sized grippers in cantilever configuration and active stabilisation. In Conference on Robotics and Automation., pages 5240-5245, Shanghai, Chine, 2011.

[26] Mokrane Boudaoud, Yassine Haddab, Yann Le Gorrec, and Philippe Lutz. Noise characterization in millimeter sized micromanipulation systems. Mechatronics, 21(6):1087-1097, 2011.

[27] E Herth, E Algré, B Legrand, and L Buchaillot. Optimization of ohmic contact and adhesion on polysilicon in mems-nems wet etching process. Microelectronic Engineering, 88(5):724-728, 2011. 